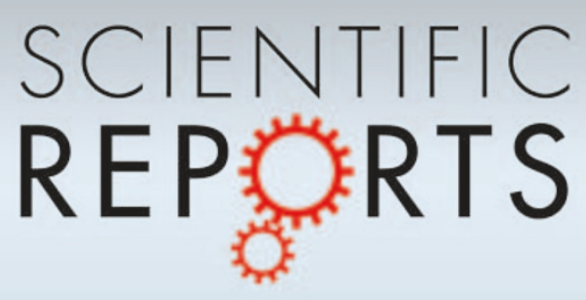

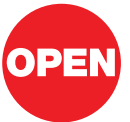

SUBJECT AREAS:

MECHANICAL

PROPERTIES

CARBON NANOTUBES AND

FULLERENES

POLYMERS AND SOFT MATERIALS

MODELLING AND THEORY

Received

25 January 2012

Accepted

21 March 2012

Published

13 April 2012

Correspondence and requests for materials should be addressed to M.T. (mut11@psu.edu) or P.M.A. (ajayan@ rice.edu)

* On leave from the Advanced Materials Department, Instituto Potosino de Investigación Científica y

Tecnológica, A.C., Camino a la Presa San José 2055, Col. Lomas 4a. sección, San Luis Potosí, SLP 78216 ,

México.

\title{
Covalently bonded three-dimensional carbon nanotube solids via boron
} induced nanojunctions

Daniel P. Hashim' ', Narayanan T. Narayanan', Jose M. Romo-Herrera², David A. Cullen³, Myung Gwan Hahm', Peter Lezzi ${ }^{4}$, Joseph R. Suttle ${ }^{5}$, Doug Kelkhoff', E. Muñoz-Sandoval7*, Sabyasachi Ganguli ${ }^{8}$, AjitK. Roy ${ }^{8}$, David J. Smith ${ }^{9}$, Robert Vajtai ${ }^{1}$, Bobby G. Sumpter ${ }^{10}$, Vincent Meunier ${ }^{11}$, Humberto Terrones ${ }^{10,12}$, Mauricio Terrones ${ }^{13}$ \& Pulickel M. Ajayan ${ }^{1}$

\begin{abstract}
${ }^{1}$ Department of Mechanical Engineering \& Materials Science, Rice University, Houston, $T X,{ }^{2}$ Departamento de Química Física and Unidad Asociada CSIC Universidade de Vigo, Campus Universitario, Vigo 3610, Spain, ${ }^{3}$ Materials Science \& Technology Division, Oak Ridge National Laboratory, Oak Ridge, TN 37831, " Department of Materials Science Engineering, Rensselaer Polytechnic Institute, Troy, NY, ${ }^{5}$ Department of Physics, Rice University, Houston, TX, ${ }^{6}$ Department of Materials Science Engineering, University of Illinois at Urbana-Champaign, ${ }^{7}$ Instituto de Microelectrónica de Madrid, IMM (CNM-CSIC), Newton 8, Tres Cantos, Spain, ${ }^{8}$ Air Force Research Laboratory, 2941 Hobson Way, WPAFB, Dayton, OH 45433-7750, USA, ${ }^{\circ}$ Department of Physics, Arizona State University, Tempe, AZ 85287, ${ }^{10}$ Center for Nanophase Materials Sciences, Oak Ridge National Lab, One Bethel Valley Road, Oak Ridge, TN 3783 1-6487, USA, ${ }^{1}$ Rensselaer Polytechnic Institute, Department of Physics, Applied Physics \& Astronomy, Troy, NY 12180 USA, ${ }^{2}$ Université Catholique de Louvain, Institute of Condensed Matter and Nanosciences, Place Croix du Sud 1 (PCPM, Boltzmann) 1348 Louvain La Neuve, Belgium, ${ }^{13}$ Department of Physics, Department of Materials Science and Engineering \& Materials Research Institute, The Pennsylvania State University, 104 Davey Lab., University Park, PA 16802-6300, USA \& Research Center for Exotic Nanocarbons (JST), Shinshu University, Wakasato 4-17-1, Nagano-city 380-8553, Japan.
\end{abstract}

The establishment of covalent junctions between carbon nanotubes (CNTs) and the modification of their straight tubular morphology are two strategies needed to successfully synthesize nanotube-based three-dimensional (3D) frameworks exhibiting superior material properties. Engineering such 3D structures in scalable synthetic processes still remains a challenge. This work pioneers the bulk synthesis of 3D macroscale nanotube elastic solids directly via a boron-doping strategy during chemical vapour deposition, which influences the formation of atomic-scale "elbow" junctions and nanotube covalent interconnections. Detailed elemental analysis revealed that the "elbow" junctions are preferred sites for excess boron atoms, indicating the role of boron and curvature in the junction formation mechanism, in agreement with our first principle theoretical calculations. Exploiting this material's ultra-light weight, super-hydrophobicity, high porosity, thermal stability, and mechanical flexibility, the strongly oleophilic sponge-like solids are demonstrated as unique reusable sorbent scaffolds able to efficiently remove oil from contaminated seawater even after repeated use.

ince the advent of carbon nanotubes (CNTs) $)^{1,2}$, engineering and controlled synthesis of these had been thoroughly investigated. Doping multi-walled (MW) and single-walled (SW) CNTs with elements such as nitrogen and boron had been used for altering their electronic properties for specific applications ${ }^{3-11}$. It was later found that dopant atoms such as nitrogen or sulfur can also induce dramatic tubule morphology changes in CNTs, including covalent multi-junctions ${ }^{12-15}$, however never were these morphologies exploited to create 3D macroscale architectures. Theoretical studies had predicted that significant structural reorganization generates stable bends in CNTs due to the presence of pentagon-heptagon defects ${ }^{16}$ that could accommodate foreign atoms besides carbon within the $\mathrm{sp}^{2}$ hybridized carbon lattice ${ }^{15}$. In addition, it was found that boron doping acts as a "surfactant" during growth to significantly increase the aspect ratio of nanotubes by preventing tube closure allowing longer tube lengths to be synthesized $(\sim 5-100 \mu \mathrm{m})$ and favoring the zigzag (or near zigzag) chirality ${ }^{17}$. Boron-doped MWCNTs $\left(\mathrm{CB}_{\mathrm{X}} \mathrm{MWNTs}\right.$ ) could be synthesized by chemical vapour deposition (CVD) using various hydrocarbons and boron sources ${ }^{18-21}$, but none of these works yielded 3D solid structures, or were able to confirm the distinct tubular morphologies induced by boron found in this article. Theoretical and experimental research had demonstrated that boron interstitial atoms located between double-walled CNTs act as atomic "fusers" or "welders" under high temperature annealing $\left(1400-1600^{\circ} \mathrm{C}\right)^{22}$, thus establishing covalent tube 
interconnections, but neither did this work produce macroscale solids. 3D solids of straight entangled non-doped CNTs were recently reported by others to create compressible sponges ${ }^{23}$ and temperature-invariant viscoelastic solids ${ }^{24}$. However neither of these works show promise towards any degree of covalent bonding established between CNTs; nor do they possess dramatic defect sites within the CNT network. An abundance of localized and topological defects, including extreme tubular morphologies, are impactful features for many applications requiring further CNT functionalization chemistry, or anchor-sites for molecular/atomic/nanoparticle adsorption (decoration) within the 3D porous solid. Furthermore, substitutionally doped CNTs provide enhanced chemical reactivity. To the best of our knowledge, this material is the first demonstration to exploit the uniqueness of heteroatom substitutional dopant effects on CNT morphology to create elastic 3D macrostructures. The combination of boron's interstitial "welding" and "surfactant" effects, makes the doping route a major step in the direction to realizing true (covalent) 3D hypothetical "superstructures" such as CNT monoliths, or interlocked nanotube ring structures recently proposed by Gogotsi $^{25}$. These architectures had been studied theoretically as fascinating future materials with superior mechanical and electrical properties $^{26}$. In this article, we describe the importance of substitutional doping effects of boron, experimentally and theoretically, so as to create a networked $\mathrm{CB}_{\mathrm{X}} \mathrm{MWNT}$ solid. We further demonstrate that these materials possess intriguing dynamic mechanical properties and can be used as a reusable oil sorbent scaffold material in seawater.

\section{Results}

The $\mathrm{CB}_{\mathrm{X}} \mathrm{MWNT}$ solids were grown directly onto the walls of a quartz tube furnace via an aerosol-assisted catalytic chemical vapour deposition (AACVD) method using triethylborane (TEB) (Aldrich >95\%) as the boron source. High mass quantities (2-3 g) of $\mathrm{CB}_{\mathrm{X}} \mathrm{MWNT}$ material was produced in just 30 minutes of growth $(60-100 \mathrm{mg} /$ min.), in the form of macroscopic elastic solids (see Supplementary Fig. S1 online), exhibiting fascinating physico-chemical properties including oleophilicity. The sample can be bent to a dramatic degree without breaking and will return to original position after released (see Fig. 1 a, b). Supplementary Movie S1 demonstrates the robust mechanical durability and flexibility in response to 'flicking' a sample by hand in a cantilever loading fashion. Remarkably, the bulk densities of the porous solids were measured to be in the range of 10 to $29 \mathrm{mg} / \mathrm{cm}^{3}$ (compared to low density carbon aerogel of $60 \mathrm{mg} / \mathrm{cm}^{3}$ ). The nanotube diameters ranged from 40 to $150 \mathrm{~nm}$, as measured from electron microscopy images (Fig. 1). The synthesized 3D architectures are entirely made up of randomly orientated and entangled CNTs with little to no amorphous carbon as depicted from SEM (Fig. 1c). The $\mathrm{X}$-ray powder diffraction pattern shows that as-produced $\mathrm{CB}_{\mathrm{X}} \mathrm{MWNT}$ sponges are indeed crystalline and showed sharp (002) diffraction peaks, but no evidence of $\mathrm{B}_{4} \mathrm{C}$ phases were found (see Supplementary Fig. S2 online). Raman spectra of the sponges are compared to those of pristine MWCNTs (see Supplementary Fig. S3 online). In this context, we noted an intense D-band, which is expected considering the contribution of boron atoms embedded in the hexagonal $\mathrm{sp}^{2}$ hybridized network of CNT sponges. Boron induces atomic-scale "elbow" junctions, as depicted in Fig. 1d and Supplementary Fig. S4 online. Many other fascinating nanotube morphologies, including covalent multi-junctions such as Y-junctions (see Supplementary Fig. S5 online) and four-way junctions (Fig. 1 e and f), were found within the entangled network structure. The most abundant of the morphologies were the stable "elbow" bends (exhibiting positive and negative curvature), which were found to be continuous and somewhat periodic along the tube length (see Supplementary Fig. S5 online). The porosity was obtained by recording the $\mathrm{N}_{2}$ gas absorption isotherm using the Brunauer-Emmett-Teller (BET) analysis technique. The results show a type-II adsorption isotherm (see Supplementary Fig.
S6 online), exhibiting a negligible concave section, which is attributed to microporous volume uptake, and a rapid rise in total volume near $\mathrm{P} / \mathrm{P}_{0}$ $=1$; a macroporous material (pore diameters $>50 \mathrm{~nm}$ ). If we assume that the density of individual MWCNTs to be around $2.1 \mathrm{~g} / \mathrm{cm}^{327}$, any sample with a density $<19 \mathrm{mg} / \mathrm{cm}^{3}$ would have a porosity $>99 \%$ (thus meaning that $>99 \%$ of the volume is air). The BET surface area, $\mathrm{S}_{\mathrm{BET}}$, was found to be $360.42 \mathrm{~m}^{2} / \mathrm{g}$ (see Supplementary Fig. S6 online).

Theoretical calculations and experimental EELS mapping of boron. The mechanism driving these stable "elbow" formations might be explained by the high stability of boron atoms on negative Gaussian curvature sites, thus present in rings with more than six carbon atoms (heptagons or octagons). In order to confirm the effect of boron on negatively curved sites, first principles calculations based on the Density Functional Theory (DFT) were carried out (using a plane wave basis code (VASP) under the GGA/PBE approximation ${ }^{28,29}$ ) to simulate doped "elbow" shape nanostructures (see boomerang-type tube in Fig. 2). A plane-wave basis with a $400 \mathrm{eV}$ energy cut-off was employed, and each structure was relaxed to $0.001 \mathrm{eV} / \mathrm{A}$ for each dopant position. According to our calculations the substitutional energy, for boron doping the boomerang-type structure, is the lowest at heptagonal rings (negative Gaussian curvature; $\mathrm{K}<0$ ), whereas nitrogen atoms are favored at the pentagonal sites (positive curvature which causes closure of the structure; $\mathrm{K}>0$ ). However, sulfur can be accommodated at both, heptagons and pentagons, thus promoting branching of multi-walled carbon nanotubes ${ }^{13,14}$. It is therefore clear that the selective preference for negative Gaussian curvature $(\mathrm{K}<0)$ of boron, and its influence in inhibiting the formation of pentagons that avoid tube closure (e.g. continuous growth of opened tubes), make boron a unique dopant which is able to catalyze the growth of these long, entangled and novel $\mathrm{CB}_{\mathrm{X}} \mathrm{MWNT}$ sponge-like structures.
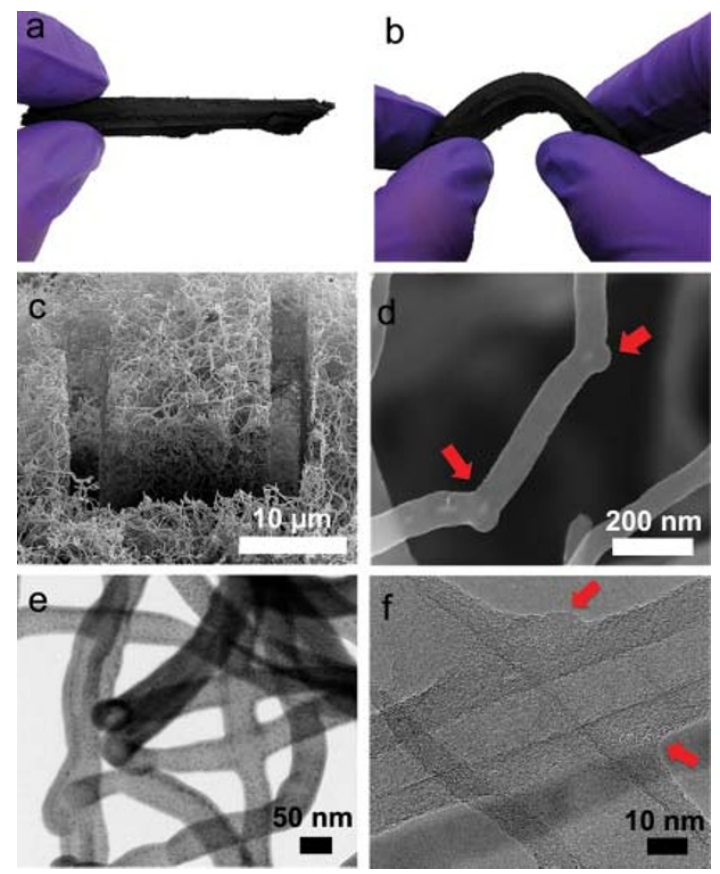

Figure 1 Physical structure and morphology of CBxMWNT 3-D network. (a) Photograph of 3D macroscopic CBxMWNT solid sponge material as-produced; (b) shows photograph of the flexibility and mechanical stablility upon bending the sample (a) by hand; (c) SEM image after ion beam "slice and view" feature showing the interior porous structure of the entangled nanotube network; (d) closer look at the "elbow" defects found in CBxMWNT solids; (e) STEM image showing two, four-way covalent nanojunctions in series; (f) TEM image showing two overlapping CBxMWNTs welded together assisted by boron doping. 


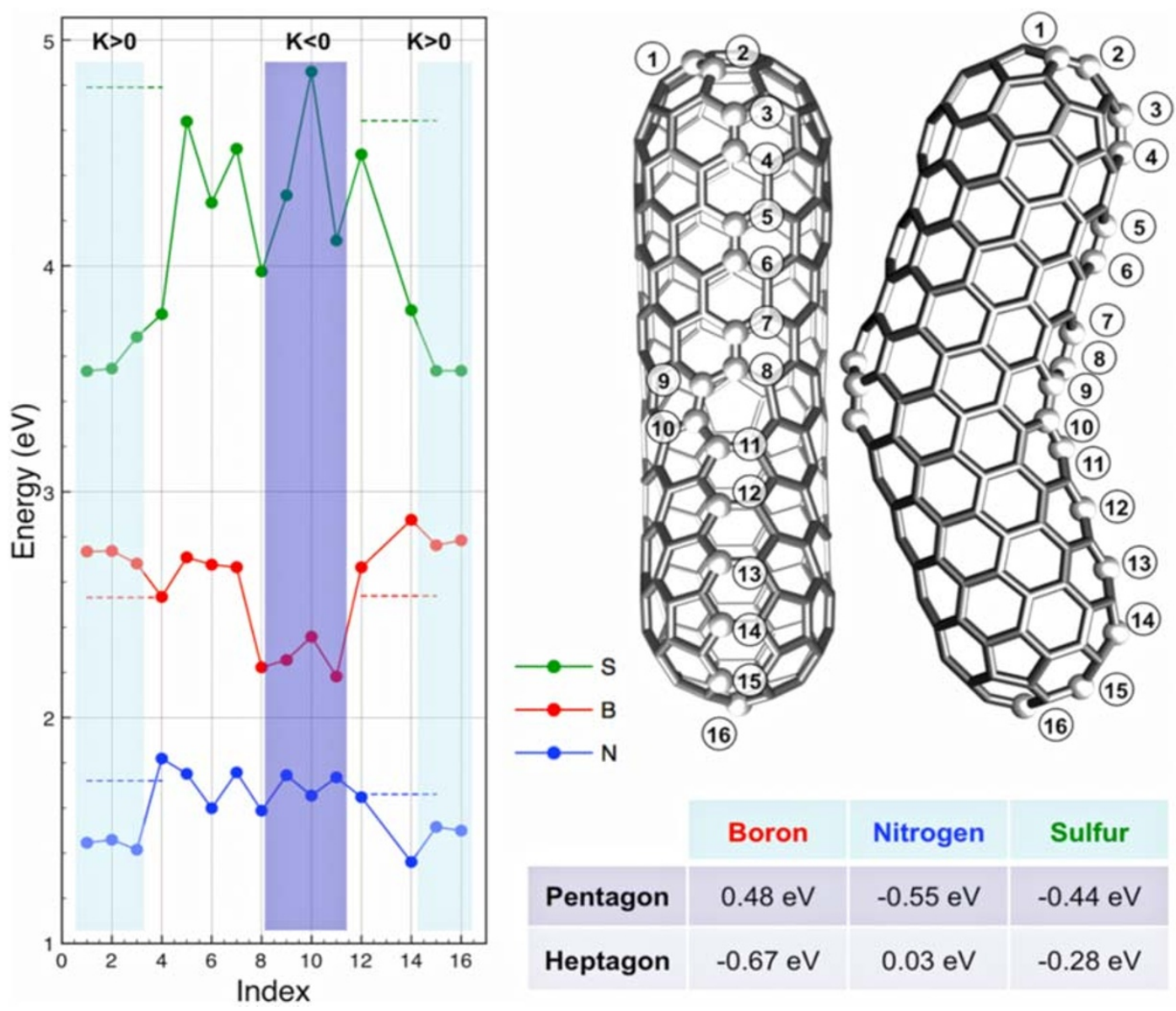

Figure $2 \mid$ Theoretical calculations using Density Functional Theory (DFT) within the GGA/PBE approximation defining stable dopant sites. Relative substitutional energies for B, N, and S dopant are shown for various positions along a $(5,5) /(9,0)$ nanotube (boomerang-type structure). The short dashed lines correspond to substitutional energy in a straight $(9,0)$ and $(5,5)$ tube, respectively. The table shows the energy average over the substitutional sites located at the pentagonal and heptagonal knee position (the energy is relative to that in a periodic $(5,5)$ nanotube). Boron does not promote any type of closure but rather strongly favors structure with a large number of regions exhibiting negative curvature.

The location of boron within the CNTs was experimentally mapped using high-angle annular-dark-field (HAADF) imaging and electron energy loss spectroscopy (EELS) line-scans using a $0.7 \mathrm{~nm}$ STEM probe. Line-scans were recorded along the edges of the tube in the region of the "elbow" defects (Fig. 3). The regions of highest $\mathrm{B}$ concentration were found to be at the location of the "elbows", suggesting that boron plays a key role in the formation of negative curvature areas inducing the formation of "elbow" junctions. Linescans across the tube diameter failed to reveal the presence of boron within the inner tube or catalyst particles, thus confirming that the B atoms were incorporated mostly within the walls of the CNTs. This trend was clearly observed in other line scans (Fig. 3c-f). Additional EELS linescans made along localized regions of high positive and negative curvature (see Supplementary Fig. S7 online) all show a stronger boron signal compared to that of the straight tube length regions and the artifact measurements from the "vacuum region". The EELS survey spectrum (see Supplementary Fig. S8 online) revealed the characteristic B and C K-shell peaks, which further confirms the presence of $B$ atoms at concentrations well within the EELS detection limit. The $\mathrm{C}$ K-edge shows maximum peaks at 287.2 and $295.4 \mathrm{eV}$ which correspond to the $1 \mathrm{~s} \pi^{*}$ and $1 \mathrm{~s}$ $\sigma^{*}$ resonance respectively. Meanwhile, the B K-edge shows maximum peaks at 193.2 and $202 \mathrm{eV}$ corresponding to the $1 \mathrm{~s} \pi^{*}$ and $1 \mathrm{~s} \sigma^{*}$ resonance respectively. The $1 \mathrm{~s} \pi^{*}$ resonance is indicative of $\mathrm{sp}^{2}$ hybridization, which indicates that boron is bonded to carbon within the carbon nanotube lattice.
X-ray photoelectron spectroscopy (XPS) data of boron (B1s) bonding state and atomic quantification. It is well known that boron doping enhances the oxidation resistance of CNTs. ${ }^{29,30}$. For this reason, the EELS elemental survey data (see Supplementary Fig. S8 online) shows higher energy boron bonding states to be dominant, thus indicating the accumulation of the glassy boron oxide layer coating the CNTs surface ${ }^{30}$. The XP spectrum revealed five underlying B1s bonding states located at peak positions 187.6, $188.8,190.1,191.9$, and $193.4 \mathrm{eV}$ marked as peaks I, II, III, IV, and $\mathrm{V}$ respectively in Fig. 3g. The lower energy peaks are associated with the $\mathrm{B}-\mathrm{C}$ bonding, such as that in $\mathrm{B}_{4} \mathrm{C}(187.8 \mathrm{eV})$, and $\mathrm{B}$-substitutedC $(188.8 \mathrm{eV})$ within the graphite crystal structure as proposed by Cermignani et $a l .^{31}$ and reported by others ${ }^{30,32-36}$. The higher energy components represent the more oxidized species corresponding to $\mathrm{BC}_{2} \mathrm{O} \quad(190.0 \mathrm{eV}), \quad \mathrm{BCO}_{2}(192.0 \mathrm{eV})$, and $\mathrm{B}_{2} \mathrm{O}_{3}(193.2 \mathrm{eV})$ respectively. It was found that the main bonding state of boron in the sponges, consist of $\mathrm{sp}^{2}$ hybridization of B-substituted-C, which supports our theoretical calculations. Quantitative elemental analysis of the boron content in this structure was revealed to be $c a .0 .7$ at $\%$.

Dynamic mechanical analysis (DMA). In addition to the formation of the $3 \mathrm{D}$ structure, the $\mathrm{CB}_{\mathrm{X}} \mathrm{MWNT}$ solid exhibit robust flexibility (Fig. 1a, b and Supplementary Movie S1 online) and good isotropic elastic mechanical behavior. At $60 \%$ compressive strain the complex modulus is $\mathrm{E}^{*}=1.26 \mathrm{MPa}$ and $\tan \delta=0.058$ (Fig. 4a). The high $\tan \delta$ value (ratio of the loss modulus E', to storage modulus E') is 

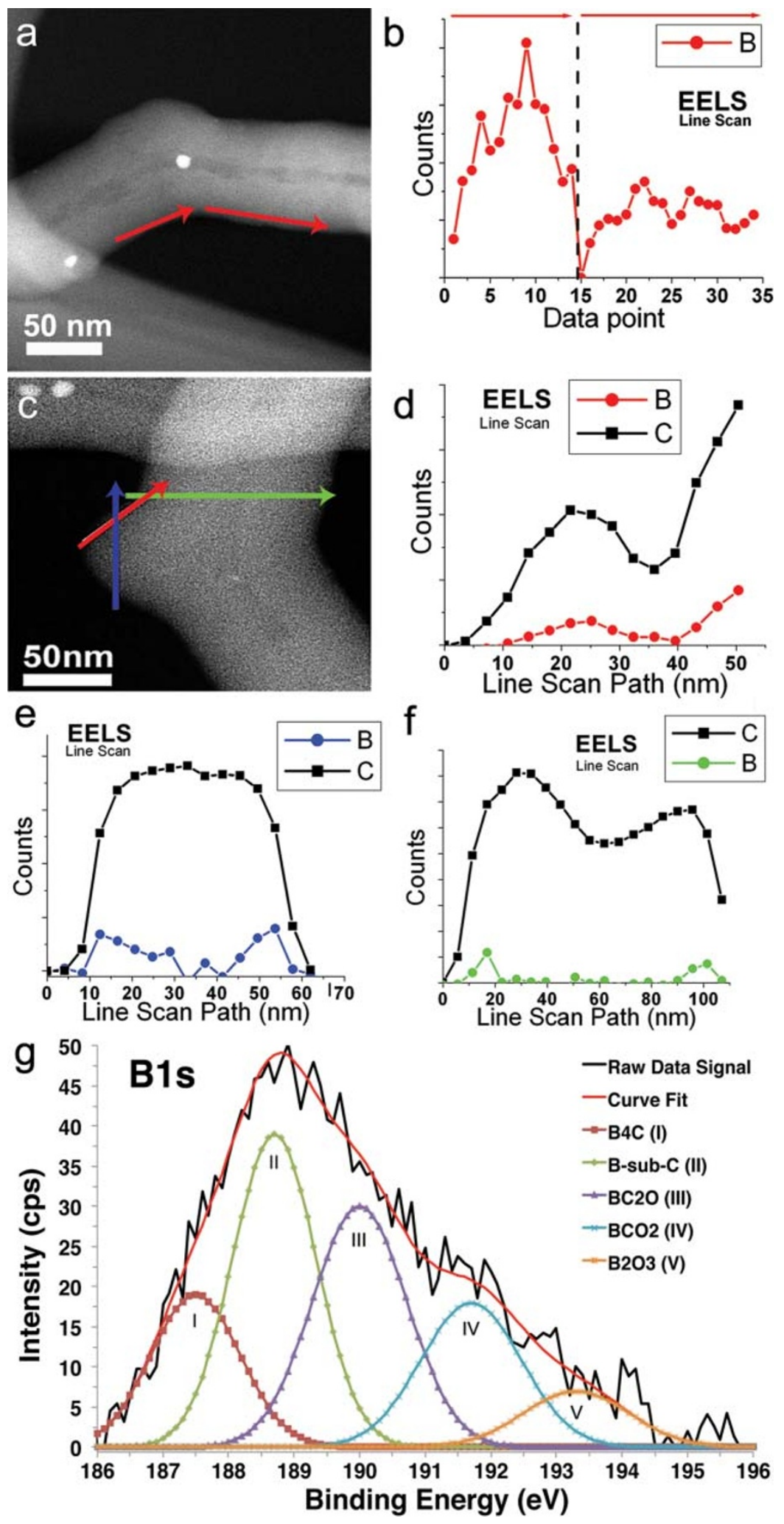

Figure 3 EELS boron mapping in regions of high curvature and XPS characterization of boron bonding state. High angle annular dark field (HAADF) images of the $\mathrm{CB}_{\mathrm{x}} \mathrm{MWNT}$ elbow defects and their corresponding EELS line scans; (a) linescan path (red arrows) along the negative curvature of elbow defect profiles for elemental boron counts; (b) along the path marked by the corresponding red arrows. The highest density of boron was found at the onset of the negative curvature; (c) HAADF image shows linescans performed on different locations (red, blue, and green arrows) mapping both elemental B (red, blue, and green) and C (black boxes) profiles; (d)-(f) seem to follow the same trend as further evidence of boron incorporation into the lattice; (f) Line scan across tube diameter (green arrow) where boron seems to be more easily detected at the outer layers near the regions of high negative curvature. The scale bar in (a) and (c) is $50 \mathrm{~nm}$; (g) XPS characterizing boron bonding states and content. Five deconvoluted B1s bonding states are revealed, after argon ion-etch, at peak positions $187.6,188.8,190.1,191.8$, and $193.4 \mathrm{eV}$ corresponding to the $\mathrm{B}-\mathrm{C}$ bonding in $\mathrm{B}_{4} \mathrm{C}(187.8 \mathrm{eV})$, $\mathrm{B}$ substituted-C within the hexagonal lattice $(188.8 \mathrm{eV}), \mathrm{BC}_{2} \mathrm{O}(190.0 \mathrm{eV}), \mathrm{BCO}_{2}(192.0 \mathrm{eV})$, and $\mathrm{B}_{2} \mathrm{O}_{3}(193.2 \mathrm{eV})$. The majority bonding state is $\mathrm{B}$ substituted-C and $\mathrm{BC}_{2} \mathrm{O}$ on the $\mathrm{CB}_{\mathrm{X}} \mathrm{MWNT}$ surface, and the boron content is $c a .0 .7 \mathrm{at} \%$. 
indicative of the materials high energy absorption and rubber-like damping capability. Due to the randomly orientated entangled CNT network, isotropic behavior is not surprising as compared to conventional anisotropic MWCNT aligned arrays as depicted in the model drawing of Figure 4b. Only $18 \%$ plastic strain deformation was observed after dynamic compression of 100 cycles at $60 \%$ strain. Further DMA results gave $\tan \delta \approx 0.11$ at $9 \%$ strain as shown in Figure $4 \mathrm{c}$. At a dynamic strain of $10 \%$ for over 11,000 cycles (Fig. $4 \mathrm{~d}$ ), a gradual increase in damping was observed, from $\tan \delta \approx 0.11$ to $\tan \delta \approx$ 0.13 , along with increasing stiffness and stress levels. These tests confirm that the structure is indeed self-intact, and a good candidate as ultra-lightweight materials with mechanical damping properties.

Oil absorbent material application. Exploiting the superhydrophobic nature of $\mathrm{CNTs}^{37}$ and the low-density 3D porous framework, the sponge-like solid is demonstrated as a reusable oil sorbent material in seawater. The $\mathrm{CB}_{\mathrm{X}} \mathrm{MWNT}$ sponge had a contact angle $c a .150^{\circ}$ with a $2 \mathrm{~mm}$ water droplet (Fig. 5a-b), but readily absorb many organic compounds and hydrocarbons, including alcohols and oils. Strong oleophilic behavior was thus observed for the sponge material with very high absorption capacity. Weight-toweight absorption capacity, (defined by $\mathrm{W}$ (wt/wt), the ratio of the final weight after absorption to the initial weight before absorption) for common solvents, was measured on sponges with three different densities: $10.8 \mathrm{mg} / \mathrm{cm}^{3}, 17.3 \mathrm{mg} / \mathrm{cm}^{3}$, and $24.3 \mathrm{mg} / \mathrm{cm}^{3}$, and plotted for a comparison (Fig. $5 \mathrm{c}$ and Supplementary Table S1 online). With $\mathrm{CB}_{\mathrm{X}} \mathrm{MWNT}$ sponges of density, $\rho=10.8 \mathrm{mg} / \mathrm{cm}^{3}$, as high as $\mathrm{W}=$ 123 for chloroform is achieved. Based on the oil absorption property, we performed the sequence of events for its possible use to clean environmental oil contamination in seawater (see Fig. 5 and Supplementary Movie S2 online). After it becomes saturated, the oil can be burned out, and the sponge can be reused in this way time and time again. The burning of the oil/solvent saturated sponge material will not destroy the $\mathrm{CB}_{\mathrm{X}} \mathrm{MWNT}$ sponge since the more volatile substance (oil/solvent) coats (protects) the CNTs' surface from significant oxidation. As the solvent/oil vanishes, the CNTs would then have less protection against oxidation, and the CNTs would only then begin to burn to a small degree before it rapidly cools below the oxidation temperature. Alternatively, the oil can be salvaged by means of squeezing it out by mechanical compression (see inset of Fig. 5f). The sponge can be used to 'mop-up' the surface oil out of the water as demonstrated by using a permanent magnet (field strength $\sim 2000 \mathrm{Oe}$ ) to move it around as a means to tracking the oil spills. The ferromagnetic properties of the sponge arise due to the iron catalyst particles used in the growth process that remains trapped in the CNT core. The room temperature magnetization curve $(\mathrm{M}(\mathrm{H}))$ (see Supplementary Fig. S9 online) indicates a very high coercive field of $\mathrm{Fe}$ catalyst nanoparticles $(\sim 400$ Oe) compared to bulk iron $(\sim 0.9$ Oe $)$. The considerable saturation magnetization and low field of saturation open up new avenues for magnetic tracking, even with small fields.

\section{Discussion}

The catalytic role of boron to prevent tube closure ${ }^{17}$ is responsible to promoting extraordinarily high yield and efficient growth kinetics for CBxMWNT production. It was found that the TEB content in the precursor had a direct relationship with the growth temperature needed for yielding the solid structure. The successful growth conditions for the sponges were very sensitive to the TEB concentration. During growth optimization, it was noticed that the presence of TEB resulted in an increase in the reaction temperature. This observation may be explained by boron atoms starting to strongly react with the iron catalyst particles to a degree that may alter the carbon diffusion,
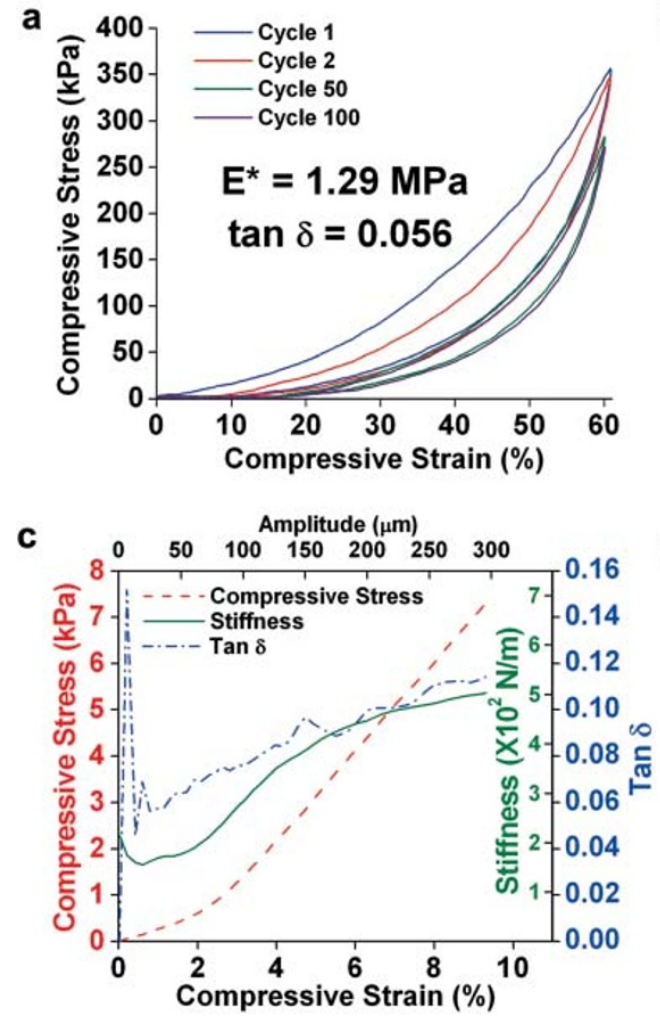

b
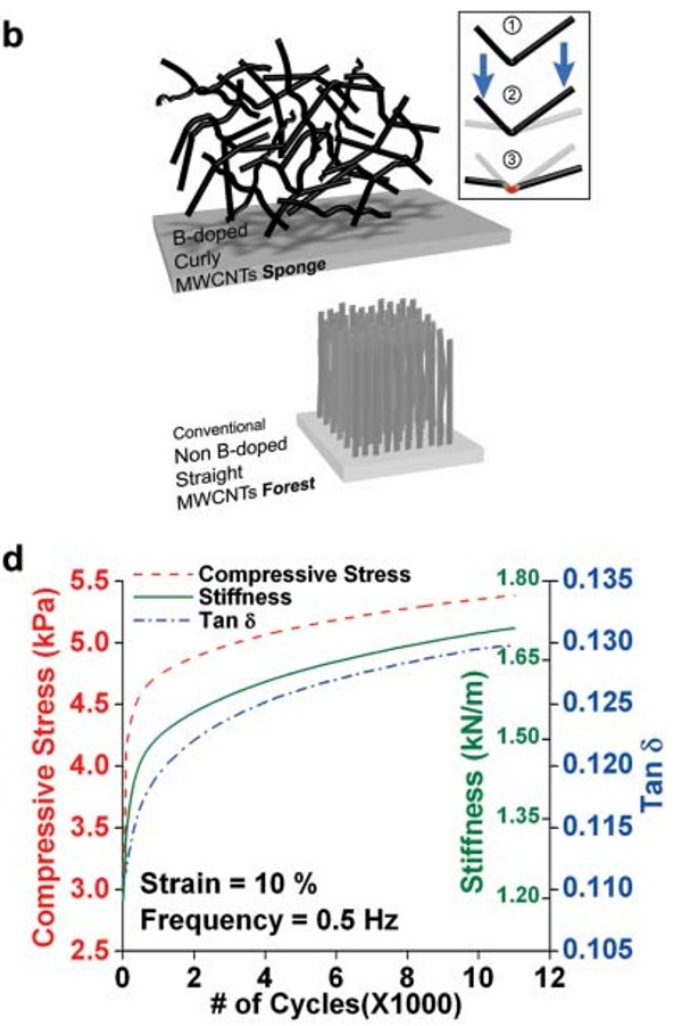

Figure $4 \mid$ Mechanical Characterization. (a) Stress-strain curves up to $60 \%$ strain at $0.5 \mathrm{~Hz}$ and room temperature for cycles 1 (blue), 2 (red), 50 (green) and 100 (purple) on a sample with $\rho=27 \mathrm{mg} / \mathrm{cm}^{3}$; (b) a computer graphics model shows the entangled random network as compared to conventional CNT arrays. The "elbow" defects aid in recovery via spring-back loading mode, shown in steps 1-3, to overcome the vdW 'sticking' force of contacting tubes upon compression which give rise energy dissipation and high tan $\delta$ values; (c) DMA on sponge with density $\approx 25 \mathrm{mg} / \mathrm{cm}^{3} \mathrm{using}$ multi-strain mode at $1 \mathrm{~Hz}$ for 250 cycles; (d) DMA on sponge with density $\approx 20 \mathrm{mg} / \mathrm{cm}^{3}$ over 11,000 cycles. 

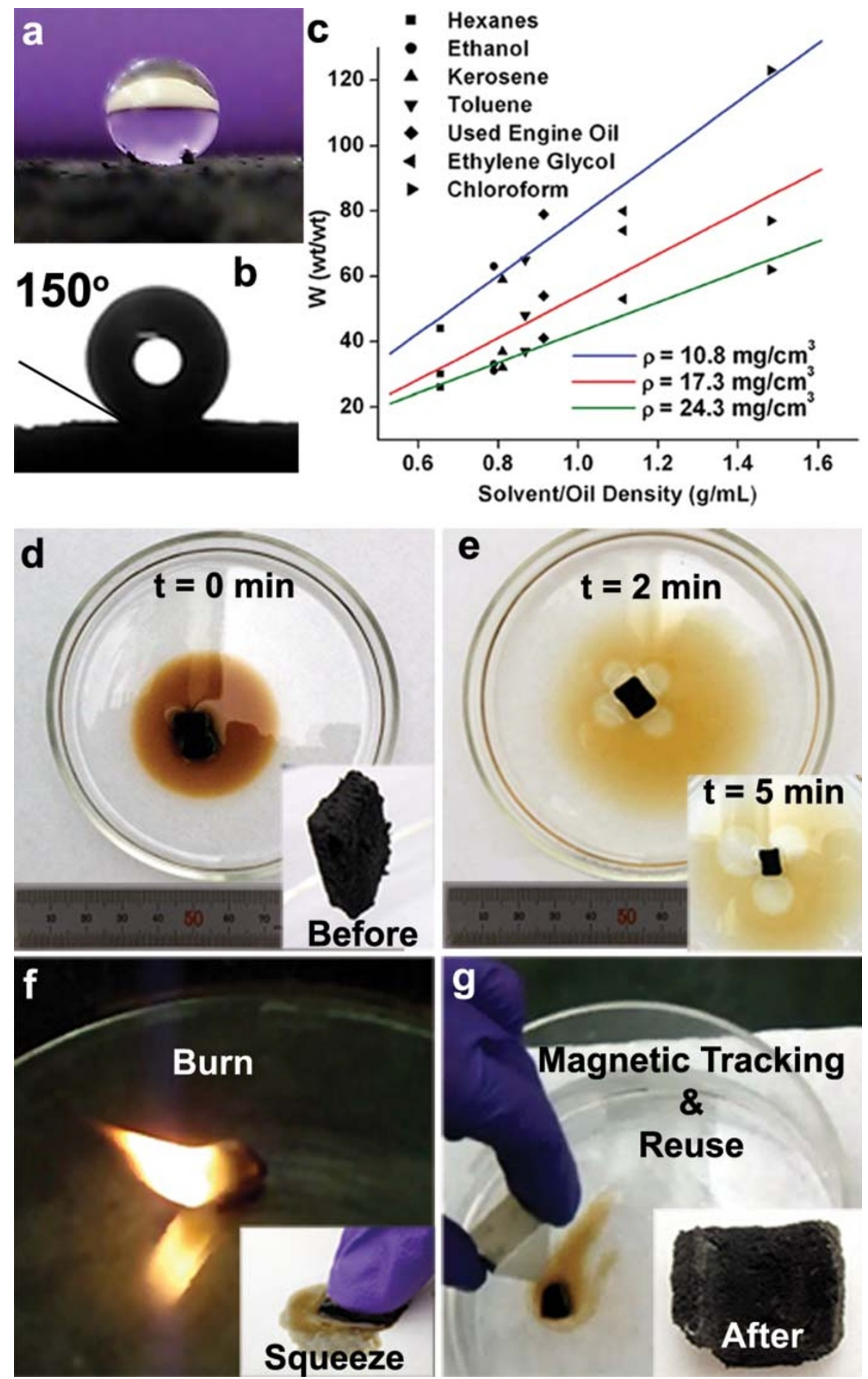

Figure 5 Oil absorption application. (a) Photograph of superhydrophobic surface with; (b) a ca. $150^{\circ}$ contact angle measurement with a 2 mm diameter water droplet resting on the sponge surface; (c) weight-to-weight (wt/wt) absorption capacity plot for common solvents measured with samples of density $24.3 \mathrm{mg} / \mathrm{cm}^{3}$ (green), $17.3 \mathrm{mg} / \mathrm{cm}^{3}$ (red), and $10.8 \mathrm{mg} / \mathrm{cm}^{3}$ (blue); (d)-(g) demonstration using CBxMWNT sponge to clean up used engine oil spill $(0.26 \mathrm{~mL})$ in seawater; sample $\left(\mathrm{m} \approx 4.8 \mathrm{mg}, \rho \approx 17 \mathrm{mg} / \mathrm{cm}^{3}\right) ;(\mathrm{d})$ photograph shows the sponge dropped into the oil at $\mathrm{t}=0 \mathrm{~min}$, inset shows sponge before use; (e) sponge sample absobing the oil at $\mathrm{t}=2 \mathrm{~min}$, inset shows $\mathrm{t}=5 \mathrm{~min}$; ( $\mathrm{f}$ ) by burning or squeezing (inset) one can salvage the oil; ( $\mathrm{g}$ ) the sponge can then be reused repetitively. By using a magnet one can track or move the oil; inset shows sponge after burning and before reuse.

saturation, and precipitation growth kinetics of long "elbowdefected" $\mathrm{CB}_{\mathrm{X}} \mathrm{MWNTs}$. It was found that the ideal Fe to $\mathrm{B}$ ratio ranges from 2 to 6 within the temperature range 900 to $850^{\circ} \mathrm{C}$ respectively. Therefore, the possible role of the catalytic effects of atomic boron on the iron catalyst particles during $\mathrm{CB}_{\mathrm{X}} \mathrm{MWNT}$ growth should not be ignored and opens opportunity for further research in order to control nanotube $3 \mathrm{D}$ architectures. Using boron as a dopant in carbon nanotube synthesis is a strategy for producing "elbows" which contribute to the elasticity of these networks. The structural integrity of the $\mathrm{CB}_{\mathrm{X}} \mathrm{MWNT} 3 \mathrm{D}$ solid foam-like material is maintained due to the boron induced defects - promoting tube-tube bonding, entanglement, and nanoscale covalent multi-junctions (see Fig. 1c-e). In this respect, the doping route seems to be more advantageous, over non-doped CNT entangled networks, holding more 
promise as a strategy to realize true (covalent) 3D solid networks with CNTs.

The DMA equipment by TA instruments was limited to stay within the perfectly elastic regime of the sponge sample, limiting our analysis to minimal strain levels. It was noticed that the higher density samples resulted in higher stress levels, as expected, being that denser samples have more network elements in the structure. Each "elbow" joint within the $\mathrm{CB}_{\mathrm{X}} \mathrm{MWNT}$ solid may act as a 'spring' joint to provide reversible elastic deformation (Fig. 4b inset). Strain amplitude on the DMA instrument was limited to the perfectly elastic regime of the material, therefore, above $10 \%$ strain, samples would start to plastically deform and the instrument would end the test as to confirm full strain recovery during testing. As a comparison, viscoelastic polymers have $\mathrm{E}^{*} \sim 20 \mathrm{MPa}$ and $\tan \delta \approx 1.0$ while most hard plastics have $\mathrm{E}^{*} \sim 1$ to $10 \mathrm{GPa}$ and $\tan \delta$ range from 0.01 to 0.10 . In a stiffness-loss map, the $\mathrm{CB}_{\mathrm{X}} \mathrm{MWNT}$ viscoelastic solids may be categorized with rubber foam. Although the mechanical data suggests the degree of covalent bonding in these solids to be fairly low, it was enough to yield perfectly self-intact elastic solids (after only 30 minutes of growth) up to $10 \%$ strains before seeing any loss in volume; and only $18 \%$ plastic strain deformation after 100 cycles at $60 \%$ strain. The increasing stiffness with sequential compression cycles may indicate some CNT alignment in the structure along the compression axis (Fig. 4d).

Highly efficient natural and synthetic sorbent materials are of current interest for environmental applications on a global scale regarding the increased risk in oil spill catastrophe. In this regard, carbon nanotube sponges are obviously superior when it comes to mass absorption efficiency. It was found that at a density of around $5 \mathrm{mg} / \mathrm{cm}^{3}$, CNT sponges are capable of reaching $\mathrm{W}=180$ for chloroform ${ }^{22}$. In comparison with woolspill ${ }^{\mathrm{TM}} \mathrm{knops}$, the leading natural oil sorbent material with a density as low as $33 \mathrm{mg} / \mathrm{cm}^{3}, \mathrm{~W}=36$ with heavy fuel oil $\left(\rho \approx 0.9535 \mathrm{~g} / \mathrm{cm}^{3}\right)^{38}$. Woolspill ${ }^{\mathrm{TM}} \mathrm{knops}$, like most natural sorbents, are hydrophilic and water uptake is expected to minimize its efficiency; therefore the super-hydrophobicity of CNT sorbent materials is a clear advantage. Interestingly, this $\mathrm{CB}_{\mathrm{X}} \mathrm{MWNT}$ solid possesses a combination of physical properties that will impact the practical use of CNTs for this application. The result of macroscopic $\mathrm{CB}_{\mathrm{X}} \mathrm{MWNT}$ solids having a network containing many covalent interconnections is a step in the right direction towards making this application more feasible, and helps to deter the drawback of environmental impact concerns of nanoscale debris. Having the ability to direct its whereabouts (oil tracking) via magnetic field (see Supplementary Movie S2 online) offers a controllable way for handling and recovering all CNT material more safely.

In summary, we report the growth of macroscale $\left(\mathrm{cm}^{3}\right.$ in size) $3 \mathrm{D}$ networked $\mathrm{CB}_{\mathrm{X}} \mathrm{MWNT}$ solids directly through an efficient largescale AACVD synthetic process. Detailed elemental analysis revealed boron to be responsible for these results and creates "elbow-like" junctions and covalent nanojunctions. These observations are in agreement with first principle calculations - indicating that the most suitable sites to host B atoms within a defective sp2-hybridized carbon network are close to heptagonal rings or negatively curved areas. These $3 \mathrm{D} \mathrm{CB}_{\mathrm{X}} \mathrm{MWNT}$ s fameworks contain many functional defectsites, which can be an advantage over its pristine counterpart. To this end, they are expected to have broad implications for many practical material applications such as selective sorbent materials, hydrogen storage $^{39,40}$, and flexible conductive scaffolds as porous $3 \mathrm{D}$ electrodes. The ultra-lightweight solid material exhibits a variety of multifunctional properties including robust elastic mechanical properties with high damping, electrical conductivity, thermal stability, high porosity, super-hydrophobicity, oleophilic behavior and strong ferromagnetism. The environmental oil removal-and-salvage application from seawater was demonstrated where the $\mathrm{CB}_{\mathrm{X}} \mathrm{MWNT}$ sponge acts as an efficient scaffold which can be controlled and recollected via a magnetically driven process, and reused multiple times.

\section{Methods}

CB $_{\mathbf{X}}$ MWNT synthesis. The aerosol-assisted chemical vapour deposition (AACVD) system was carried out under atmospheric pressure conditions and comprises a horizontal hot-wall quartz tube reactor chamber heated by a furnace $(30 \mathrm{~cm}$ heating zone). Solutions were prepared mixing toluene (Aldrich, anhydrous, 99.8\%) and ferrocene $\left(\mathrm{Fe}\left(\mathrm{C}_{5} \mathrm{H}_{5}\right)_{2}\right)$ (Alpha Aecer $\left.99 \%\right)$ at a concentration of $25 \mathrm{mg} / \mathrm{mL}$, and triethylborane (TEB) $\left(\left(\mathrm{C}_{2} \mathrm{H}_{5}\right)_{3} \mathrm{~B}\right)$ (Aldrich $\left.>95 \%\right)$ at Fe:B ratio 5:1, followed by 30 minute sonication. The TEB was added while in a glove box under an inert nitrogen atmosphere. The precursor solution was placed in a glass vessel with an ultrasonic piezoelectric transducer film (diameter $=40 \mathrm{~mm}$ ) at the bottom (Pyrosol 7901 type manufactured by RBI instrumentation). The piezoelectric frequency and amplitude was controlled by an external generator source providing a resonant frequency at $0.8 \mathrm{MHz}$. The solution feed rate was varied 0.4 to $0.8 \mathrm{ml} / \mathrm{min}$ for a total synthesis time of 30 minutes. The aerosol, or micro-droplet size mist cloud, generated above the solution was transferred into the reactor chamber by the argon carrier gas at a flow rate of $2.50 \mathrm{~L} / \mathrm{min}$. The furnace temperature was $860^{\circ} \mathrm{C}$ in the chamber reactor zone where the precursor solution was vaporized. Deposition and growth occurred directly onto the 1 inch diameter quartz tube walls taking on the shape of the tube (see Supplementary Fig. S1 Online) yielding between 2-3 grams of CBxMWNT material per 30 minute synthesis $(66-100 \mathrm{mg} / \mathrm{min})$. Control of the density and overall stiffness (resilience) of the sponge is achievable by changing the precursor solution feed rate during growth; lower feed rate yielded a lower density material that was more soft and flexible.

Microscopy characterization. The morphology and structural properties were extensively studied by SEM (FEI-field emission SEM - XL30 operated at 1-15 keV), TEM/STEM (JEOL 2010 F instrument equipped with a Gatan Enfina energy-loss spectrometer.

XPS characterization. The chemical bonding states and atomic quantification of boron content within the CBxMWNT solids were studied using a Phi Quantera instrument equipped with monochromatic $\mathrm{Al}(\mathrm{K}-\alpha) 1486.6 \mathrm{eV}$ X-ray source at $50 \mathrm{~W}$ and a $200-\mu \mathrm{m}$-beam diameter. Argon ion-etch pre-treatment was performed for 2 minutes using a $3 \mathrm{kV}$ beam and target emission current at $7 \mathrm{~mA}$. Survey scans were performed at $140 \mathrm{eV}$ pass energy, and $\mathrm{C} 1 \mathrm{~s}$ and $\mathrm{B} 1 \mathrm{~s}$ elemental scans at $55 \mathrm{eV}$ pass energy with $0.10 \mathrm{eV}$ steps. Data analysis software was used for the peak fitting using Guassian functions and a linear baseline. All peaks were generated having FWHM limited to $<2.0 \mathrm{eV}$. Our fits provided values of chi-squared $=8.127$ and $\mathrm{R}^{2}=0.978$ Before fitting, the background was subtracted using the software and the peaks were calibrated to the $\mathrm{C} 1 \mathrm{~s}$ peak located at $184.6 \mathrm{eV}$ for graphitic carbon.

Mechanical characterization. Dynamic mechanical tests at $60 \%$ strain were performed on samples with density $\approx 27 \mathrm{mg} / \mathrm{cm}^{3}$ using an Intstron Electropuls E3000 instrument and Wavematrix software. For the lower strain amplitude tests at $\sim 9 \%$ strain, TA instruments DMA model Q800 was used. Tan $\delta$ values (the ratio of loss modulus, E”, to storage modulus, E') and sample stiffness data were measured from a sponge block with density $\approx 25 \mathrm{mg} / \mathrm{cm}^{3}$ and size: $2.4015 \mathrm{~mm} \times 11.2167 \mathrm{~mm}^{2}$ under compression tests in multi-strain mode with amplitudes ranging 0-300 $\mu \mathrm{m}$ (corresponding to strains up to $\sim 9 \%$ ) at a frequency of $1 \mathrm{~Hz}$ and $0.01 \mathrm{~N}$ preload force. A total of 50 data points were collected from 250 cycles to make the plot of Fig. $4 c$, with each sequential cycle and data point having a linear increase in strain amplitude from $0-300 \mu \mathrm{m}$ (corresponding to strains of $0-9 \%$ ).

Oil/Solvent absorption capacity measurements. The absorption capacity values, $\mathrm{W}$ $(\mathrm{wt} / \mathrm{wt})$, were obtained by measuring the mass of the dry as-produced sponge, and then measuring the mass after oil/solvent absorption. The ratio of the final mass to the initial mass was taken as the $\mathrm{W}$ (wt/wt) value, averaging out three samples. To ensure full saturation was obtained before weighing, the samples were left submerged in the solvent/oil (without water) overnight. The samples were then removed with sharp needle tweezers and immediately placed onto a weigh paper to be measured on the mass balance.

1. Iijima, S. Helical Microtubules of Graphitic Carbon. Nature 354, 56-58 (1991)

2. Oberlin, A., Endo, M. \& Koyama, T. Filamentous growth of carbon through benzene decomposition. J. Cryst Growth 32, 335-349 (1976).

3. Stephan, O. et al. Doping graphitic and carbon nanotube structures with boron and nitrogen. Science 266, 1683-1685 (1994).

4. Suenaga. K. Synthesis of nanoparticles and nanotubes with well-separated layers of boron nitride and carbon. Science 278, 653-655 (1997).

5. Terrones, M. et al. Metal particle catalysed production of nanoscale BN structures. Chem. Phys. Lett. 257, 573-576 (1996).

6. Terrones, M. et al. Efficient route to large arrays of $\mathrm{CN}_{\mathrm{x}}$ nanofibers by pyrolysis of ferrocene/melamine mixtures. Appl. Phys. Lett. 75, 3932-3935 (1999).

7. Yudasaka, M., Kikuchi, R., Ohki, Y. \& Yoshimura, S. Nitrogen-containig carbon nanotube growth from Ni phthalocyanine by chemical vapor deposition. Carbon 35, 195-201 (1997).

8. Suenaga, K., Yudasaka, M., Colliex, C. \& Iijima, S. Radially modulated nitrogen distribution in $\mathrm{CN}_{\mathrm{x}}$ nanotubular structures prepared by CVD using $\mathrm{Ni}$ phthalocyanine. Chem. Phys. Lett. 316, 365-372 (2000).

9. Wang, X. et al. Controlled Growth, Structure, and Low Field Emission of WellAligned $\mathrm{CN}_{\mathrm{X}}$ Nanotubes. J. Phys. Chem. B 106, 2186-2190 (2002). 
10. Ma, X. et al. Polymerized carbon nanobells and their field emission properties. Appl. Phys. Lett. 75, 3105-3108 (1999).

11. Redlich, $\mathrm{Ph}$. et al. B-C-N nanotubes and boron doping of carbon nanotubes. Chem. Phys. Lett. 260, 465-470 (1996).

12. Sumpter, B. G. et al. Nitrogen-Mediated Carbon Nanotube Growth: Diameter Reduction, Metallicity, Bundle Dispersability, and Bamboo-like Structure Formation. ACSNano 1, 369-375 (2007).

13. Romo-Herrera, J, M. et al. An atomistic branching mechanism for carbon nanotubes: Sulfur as the triggering agent. Angewandte Chemie 47, 2948-2953 (2008).

14. Romo-Herrera, J. M. et al. The Role of Sulfur in the synthesis of Novel Carbon Morphologies: From Covalent Y-Junctions to Sea-Urchin-Like Structures. Adv. Func. Mater. 19, 1193-1199 (2009).

15. Sumpter, B. G. et al. A Theoretical and Experimental Study On Manipulating the Structure and Properties of Carbon Nanotubes Using Substitutional Dopants. Int. J. Quantum Chem. 109, 97-118 (2009).

16. Dunlap, B. I. Connecting carbon tubules. Phys. Rev. B 46, 1933-1936 (1992).

17. Blase, X. et al. Boron-Mediated Growth of Long Helicity-Selected Carbon Nanotubes. Phys. Rev. Lett. 83, 5078-5081 (1999).

18. Mondal, K. C., Coville, N. J., Witcomb, M. J., Tejral, G. \& Havel, J. Boron mediated synthesis of multiwalled carbon nanotubes by chemical vapor deposition. Chem. Phys. Lett. 437, 87-89 (2007).

19. Lozano-Castello, D. et al. Preparation and characterisation of novel "seacucumber"-like structures containing carbon and boron. Carbon 42, 2223-2231 (2004).

20. Koós, A. A., Dillon, F., Obraztsova, E. A., Crossley, A. \& Grobert, N. Comparison of Structural changes in nitrogen and boron-doped multi-walled carbon nanotubes. Carbon 48, 3033-3041 (2010).

21. Lyu, S. C., Han, J. H., Shin, K. W. \& Sok, J. H. Synthesis of boron-doped doublewalled carbon nanotubes by the catalytic decomposition of tetrahydrofuran and triisopropylborate. Carbon 49, 1532-1541 (2011).

22. Endo, M. et al. Atomic Nanotube Welders: Boron Interstitials Triggering Connections in Double-Walled Carbon Nanotubes. Nano Lett. 5, 1099-1105 (2005).

23. Gui, X. et al. Carbon Nanotube Sponges. Adv. Mater. 22, 617-621 (2010).

24. Xu, M., Futaba, D. N., Yamada, T., Yumura, M. \& Hata, K. Carbon Nanotubes with Temperature-Invariant Viscoelasticity from $-196^{\circ}$ to $1000^{\circ} \mathrm{C}$. Science 330, 1364-1368 (2010)

25. Gogotsi, Y. High-Temperature Rubber Made from Carbon Nanotubes. Science 330, 1332-1333 (2010).

26. Romo-Herrera, J. M., Terrones, M., Terrones, H., Dag, S. \& Meunier, V. Covalent 2D and 3D networks from 1D nanostructures: designing new materials. Nano Lett. 7, 570-576 (2007).

27. Lehman, J. H., Terrones, M., Mansfield, E., Hurst, K. E. \& Meunier, V. Evaluating the characteristics of multiwall carbon nanotubes. Carbon 49, 2581-2602 (2011).

28. Kresse, G., \& Furthmuller, J. Efficient iterative schemes for ab initio total-energy calculations using a plane-wave basis set. Phys. Rev. B 54, 11169-11186 (1996).

29. Perdew, J. P., Burke, K. \& Ernzerhof, M. Generalized gradient approximation made simple. Phys. Rev. Lett. 77, 3865-3868 (1996).

30. Yang, L. et al. Boron-doped carbon nanotubes as metal-free electrocatalysts for the oxygen reduction reaction. Angewandte Chemie-International Ed. 50, 7132-7135 (2011)

31. Wu, X. \& Radovic, L. R. Inhibition of catalytic oxidation of carbon/carbon composites by boron-doping. Carbon 43, 1768-1777 (2005).

32. Cermignani, W., Paulson, T. E., Onneby, C. \& Pantano, C. G. Synthesis and characterization of boron doped carbons. Carbon 33, 367-374 (1995).

33. Liu, Y. S., Zhang, L. T., Cheng, L. F., Yang, W. B. \& Xu, Y. D. Effect of deposition temperature on boron-doped carbon coatings deposited from a $\mathrm{BCl}_{3}-\mathrm{C}_{3} \mathrm{H}_{6}-\mathrm{H}_{2}$ mixture using low pressure chemical vapor deposition. Appl. Surf. Sci. 255, 87618768 (2009).

34. Jacobsohn, L. G., Schulze, R. K., Maia da Costa, M. E. H. \& Nastasi, M. X-ray photoelectron spectroscopy investigation of boron carbide films deposited by sputtering. Surf. Sci. 572, 418-424 (2004).
35. Shirasaki, T., Derre, A., Menetrier, M., Tressaud, A. \& Flandrois, S. Synthesis and characterization of boron-substituted carbons. Carbon 38, 1461-1467 (2000).

36. Burgess, J. S. et al. Boron-doped carbon powders formed at $1000^{\circ} \mathrm{C}$ and one atmosphere. Carbon 46, 1711-1717 (2008).

37. Li, S. et al. Super-Hydrophobicity of Large-Area Honeycomb-Like Aligned Carbon Nanotubes. J. Phys. Chem. B 106, 9274-9276 (2002).

38. McFarlane, I. D., Hamilton, M. A. \& Carnaby, G. A. Absorbent materials and use thereof, USPTO Patent No. 5,252,215, (1993).

39. Singh, A. K., Sadrzadeh, A. \& Yakobson, B. Metallacarboranes: Towards Promising Hydrogen Storage Metal Organic Frameworks. J. Am. Chem. Soc. 132, 14126-14129 (2010)

40. Froudakis, G. E. Hydrogen storage in nanotubes \& nanostructures Materialstoday 14, 324-328 (2011).

\section{Acknowledgement}

This material is based upon work supported by the National Science Foundation Graduate Research Fellowship under Grant No. 0940902 awarded to D. P. Hashim. P.M. Ajayan, M. Terrones, and N. T. Narayanan acknowledge funding sponsorship from the DOD: Air Force Office of Scientific Research for the Project MURI: Synthesis and Characterization of 3D Carbon Nanotube Solid Networks Award No.: FA9550-12-1-0035, M.G. Hahm, and R.Vajtai acknowledge financial support from ARL/ARO (No.W911NF). Special thanks to Daniel Ramírez-Gonzalez for technical support. The authors gratefully acknowledge use of facilities within the John M. Cowley Center for High Resolution Electron Microscopy at Arizona State University. H. Terrones acknowledges support as visiting professor from the Ecole Polytechnique of Louvain, and of the Center for Nanophase Materials Science (CNMS) of Oak Ridge National Laboratory. M. Terrones thanks JST-Japan for funding the Research Center for Exotic NanoCarbons, under the Japanese regional Innovation Strategy Program by the Excellence. B. G. Sumpter was supported by the Center for Nanophase Materials Sciences, which is sponsored by the Office of Basic Energy Sciences at Oak Ridge National Laboratory, U.S. Department of Energy. Some of the calculations were performed using resources of the Oak Ridge Leadership Computing Facility and the National Center for Computational Sciences. V.M. was supported in part by the New York State under NYSTAR contract C080117.

\section{Author contributions}

D.P. H. originated the project ideas. D. P. H., P. L., J. R. S., and D. K. worked together as team of undergraduate students for synthesis of CBxMWNTs. J. M. R. provided some SEM images and figure drawing. D. A. C. and D. J. S. performed EELS. D.P.H. performed the XPS analysis. D. P. H., N. T. N. performed oil absorption experiments. N.T.N. performed the BET analysis. M. G. provided the Raman spectroscopy and helped prepare manuscript. E. M-S. and N. T. N. provided magnetization data. S. G. and A. K. R. provided TEM images B. G. S. and V. M. made first principles theoretical calculation. P. M. A., R. V., M. T., and H. T. were supervisors responsible for the project planning and co-wrote the paper with D. P. H. All the authors discussed the results.

\section{Additional information}

Supplementary information accompanies this paper at http://www.nature.com/ scientificreports

Competing financial interest: The authors declare no competing financial interests.

License: This work is licensed under a Creative Commons

Attribution-NonCommercial-ShareAlike 3.0 Unported License. To view a copy of this license, visit http://creativecommons.org/licenses/by-nc-sa/3.0/

How to cite this article: Hashim, D.P. et al. Covalently bonded three-dimensional carbon nanotube solids via boron induced nanojunctions. Sci. Rep. 2, 363; DOI:10.1038/srep00363 (2012) 